\title{
Notes on taxonomy and distribution of some Ortochile Latreille, 1809, Sybistroma Meigen, 1824 and Teuchophorus Loew, 1857 (Diptera: Dolichopodidae) species from Mediterranean Region
}

\section{Заметки о систематике и распространении некоторых видов Ortochile Latreille, 1809, Sybistroma Meigen, 1824 и Teuchopborus Loew, 1857 (Diptera: Dolichopodidae) Средиземноморья}

\author{
I.Ya. Grichanov ${ }^{1 *}, M$. Nourti ${ }^{2}$ \\ И.Я. Гричанов ${ }^{1 *}$, М. Ноурти ${ }^{2}$
}

\footnotetext{
${ }^{1}$ All-Russian Institute of Plant Protection, Podbelskiy roadway, 3, St. Petersburg-Pushkin 196608 Russia. E-mail: grichanov@mail.ru

${ }^{1}$ Всероссийский НИИ защиты растений, ш. Подбельского, 3, Санкт-Петербург, Пушкин 196608, Россия.

2 Faculté des Sciences, Université Abdelmalek Essaâdi, Tétouan, Morocco; E-mail: nourti.mohamed2@gmail.com

*corresponding author.
} key.

KEY WORDS: Long-legged flies, Palaearctic Region, new record, new combination, new name, new synonym,

КЛЮЧЕВЫЕ СЛОВА: Мухи-зеленушки, Палеарктика, новое указание, новая комбинация, новое название, новый синоним, ключ.

ABSTRACT. New data on some Mediterranean dolichopodid species are discussed. The following re-combinations (from Hercostomus) are proposed in the paper (comb.n.): Ortochile morenae (Strobl, 1899), Sybistroma parvilamellata (Macquart, 1827), Sybistroma parvula (Parent, 1927), Sybistroma quadrifilata (Strobl, 1899) and Sybistroma quadrifilata (Becker, 1917, nec Strobl, 1899). Gymnopternus quadrifilatus Strobl, 1899 is removed from synonymy with Sybistroma parvilamellata and placed in synonymy to Sybistroma parvula (syn.n.). A new name Sybistroma theodori Grichanov et Nourti, nom.n., is proposed for Hercostomus quadrifilatus Becker, 1917 (nec Strobl, 1899). A neotype for Hercostomus quadrifilatus Becker is established. Updated keys to known species of Ortochile and Mediterranean species of Sybistroma are compiled. Sybistroma parvula and $S$. theodori are found in Morocco for the first time. Teuchophorus rifensis Nourti, Grichanov et Kettani, 2019 described from Morocco is found in earlier reports from Algeria and Krasnodar Territory of Russia under the name T. bipilosus Becker, 1908. The latter species is removed from faunas of Algeria and Russia.

РЕЗЮМЕ. Обсуждаются новые данные по некоторым видам средиземноморских мух-зеленушек. В статье предлагаются следующие рекомбинации (из рода Hercostomus) (comb.n.): Ortochile morenae (Strobl, 1899), Sybistroma parvilamellata (Macquart,
1827), Sybistroma parvula (Parent, 1927), Sybistroma quadrifilata (Strobl, 1899) и Sybistroma quadrifilata (Becker, 1917, nec Strobl, 1899). Gymnopternus quadrifilatus Strobl, 1899 исключён из синонимии с Sybistroma parvilamellata и помещён в синонимы к Sybistroma parvula (syn.n.). Новое название, Sybistroma theodori Grichanov et Nourti, nom.n., предложено для Hercostomus quadrifilatus Becker, 1917 (nec Strobl, 1899). Выделен неотип Hercostomus quadrifilatus Becker. Составлены обновлённые определители известных видов Ortochile и средиземноморских видов Sybistroma. Sybistroma parvula и $S$. theodori впервые обнаружены в Марокко. Teuchophorus rifensis Nourti, Grichanov et Kettani, 2019, описанный из Марокко, обнаружен среди ранее опубликованных указаний из Алжира и Краснодарского края России под названием T. bipilosus Becker, 1908. Последний вид исключён из фаун Алжира и России.

\section{Introduction}

The Moroccan fauna of Dolichopodidae is largely understudied, with 110 previously known species [Kawtar Kettani, pers. comm.]. Recent field work in different Moroccan areas has uncovered some rare species that need revision of their status. The aim of this article is to contribute to a better knowledge of this fauna by providing new records of poorly known species and taxonom-

How to cite this article: Grichanov I.Ya., Nourti M. 2021. Notes on taxonomy and distribution of some Ortochile Latreille, 1809, Sybistroma Meigen, 1824 and Teuchophorus Loew, 1857 (Diptera: Dolichopodidae) species from Mediterranean Region // Russian Entomol. J. Vol.30. No.2. P.189-195. doi: 10.15298/rusentj.30.2.14 
ical notes on rare species of the genera Ortochile Latreille, 1809, Sybistroma Meigen, 1824 and Teuchophorus Loew, 1857. New combinations, a new name and a new synonym are established. Updated keys to known species of Ortochile and Mediterranean species of Sybistroma are compiled.

\section{Material and methods}

The new material was collected at the north of Morocco from the Rif Mountains with entomological sweep net during the winter of 2020. The specimens in ethanol were studied and photographed with a ZEISS Discovery V-12 stereo microscope and an AxioCam MRc5 camera. Then they were dried from ethanol and mounted on pin. Morphological terminology and abbreviations follow Cumming, Wood [2017] and Grichanov, Brooks [2017]. Material cited in this work will be housed at the Zoological Institute of the Russian Academy of Sciences (ZIN, St. Petersburg, Russia).

\section{Ortochile morenae (Strobl, 1899), comb.n.} Figs 1-2, 6.

Gymnopternus morenae Strobl, 1899: 119 [as Gymnopternus (Dasyarthrus) Morenae]. Type locality: Spain: Sierra Morena. Dasyarthrus morenae: Becker, 1917: 225.

Hercostomus morenae: Becker, 1917: 225; Nourti et al., 2019a: 124

MATERIAL EXAMINED. $1 \sigma^{7}$, 19 , Morocco: Fahs-Anjra,

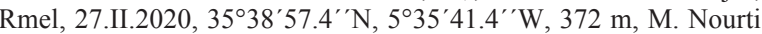
leg.

HABITAT. The same as that of Sybistroma parvula (Parent, 1927) (see below).

NOTES. The species was described from the Sierra Morena mountain range in southern Spain and was probably never reported again until recently. Outside Spain, it was found only in Morocco, on river banks, marshes and dune marshes in Rif Mountains [Nourti et al., 2019a]. A thorough investigation of the newly collected material has shown that the species morphology corresponds entirely with the generic concept of Ortochile as described by Brooks [2005]. It has proboscis as long as head is high; vein $\mathrm{M}$ beyond crossvein dm-m with weak anterior bend before middle, convergent with $\mathrm{R}_{4+5}$ and ending well above wing apex, close to apex of $\mathrm{R}_{4+5}$. O. morenae is the fourth known species of the genus and the second species in Morocco. At present it is distributed in Morocco and Spain.

DIAGNOSIS. Ortochile morenae is close to O. nigrocoerulea Latreille, 1809, differing in shorter proboscis, at most as long as height of head in male, broad palpus, morphology of hypopygium (see key below). The synonymy of O. nigrocoerulea and O. unicolor Loew, 1850 [Yang et al., 2006; Grichanov, 2007] needs confirmation.

\section{Key to THE SPECIES OF ORTOCHILE (MAles)}

This key is built on Parent [1938] and Stackelberg [1941] but has modifications based on material examined for the present study. The species distribution follows Yang et al. [2006].
1. Fore coxa with white hairs, long and dense; femora mainly yellow, with hind femur black at apex; lower postocular setae multiserial; hind tarsus and three middle segments of mid tarsus distinctly thickened; body length 4-5 mm (Spain) ........................... O. barbicoxa Strobl, 1909

- Fore coxa with black hairs, short and sparse; femora mainly or entirely black; lower postocular setae uniserial; tarsi simple or differently modified ......................... 2

2. Mid tarsus with segments 1-4 yellow, long and thin, segment 5 black, flattened and widened; body length 3-4 mm (France, Italy, Malta) ....... O. soccata Loew, 1850

- Mid tarsus simple, entirely black ........................... 3

3. Legs entirely black; proboscis at most as long as height of head; epandrial lobe thin; body length $3 \mathrm{~mm}$ (Morocco, Spain) ............................ O. morenae (Strobl, 1899)

- At least tibiae mainly or entirely yellow; proboscis about 2 times as long as height of head; epandrial lobe broad, clavate; body length 3-5 mm (West and South Europe, North Africa, Middle East)....

O. nigrocoerulea Latreille, 1809; O. unicolor Loew, 1850

\section{Sybistroma parvilamellata (Macquart, 1827), comb.n.}

Dolichopus parvilamellatus Macquart, 1827: 66. Type locality: not given [North France].

Hercostomus parvilamellatus: Bezzi, 1903: 312.

Gymnopternus parvilamellatus: Loew, 1857: 18.

NOTES. Studying the type material from Strobl's collection, Morge and Negrobov [1981] placed Gymnopternus (Hypophyllus) quadrifilatus Strobl, 1899 in synonymy with Hercostomus parvilamellatus. Nevertheless, figures provided by the authors and our material examined (see below) have shown that the name G. quadrifilatus is a synonym of Hercostomus parvulus Parent, 1927. The latter species is very close to $H$. parvilamellatus, but well differing in characters provided by Parent [1938]. The morphology of both species corresponds entirely with the generic concept of Sybistroma as described by Brooks [2005]. Their males are lacking modified antennae, but can be distinguished by the possession of greatly elongated and setose apicoventral epandrial lobes; the antenna has enlarged scape, reduced pedicel; the wing has weak sinuous anterior bend before middle; the hypopygium has basiventral epandrial lobes elongate and digitiform, shifted ventrally and lying beside hypandrium. Parent [1938] included "Russia" into the area of the species. However, we do not know papers with original material from this country. $S$. parvilamellata was reported from Belgium, France, Germany, Italy, Spain and UK.

DIAGNOSIS. S. parvilamellata and S. parvula are close to $S$. lorifera (Mik, 1878), differing from the latter in nonthickened antennal stylus and relatively short apicoventral epandrial lobe. S. parvilamellata differs from S. parvula in antennal stylus with basal segment slightly shorter than apical segment; cercus elbowed, yellow, with long apical setae; phallus expanded distally (see key below).

\section{Sybistroma parvula (Parent, 1927), comb.n.}

$$
\text { Figs 3-4, 8-9. }
$$

Hercostomus parvulus Parent, 1927: 228, 229 (in key) (described in Parent, 1928: 8). Type locality: Spain: Algeciras.

Gymnopternus quadrifilatus Strobl, 1899: 120 [as Gymnopter-

Figs 1-5. Habitus: 1-2 - Ortochile morenae; 3-4 - Sybistroma parvula; 5 - Sybistroma theodori; 1, 3, 5- males; 2, 4 - females. Photographs by I.Ya. Grichanov.

Рис. 1-5. Внешний вид: 1-2 - Ortochile morenae; 3-4 - Sybistroma parvula; 5 -Sybistroma theodori; 1, 3, 5 - caмцы; 2, 4 самки. Фото И.Я. Гричанова. 


nus (Hypophyllus) quadrifilatus], syn.n. Type locality: Spain: Algeciras.

Hypophyllus quadrifilatus: Bezzi, 1903: 314.

Hercostomus quadrifilatus: Becker, 1917: 234; Morge, Negrobov, 1981: 13 (as synonym of Hercostomus parvilamellatus).

Sybistroma quadrifilata (Strobl, 1899), comb.n.

MATERIAL EXAMINED. $20^{7}, 2+$, Morocco: Fahs-Anjra, Rmel,

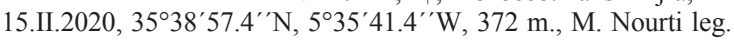

HABITAT. Represents a scrubland with rocky soil of a clay-calcareous nature crossed by a small stream and bordered by vegetation mainly composed of Pistacia lentiscus L., Carlina racemosa L., Conyza conodensis L., Erica arborea L., and Cistus monspeliensis L. Further afield, fruit trees grow, such as Olea europea L. and Ficus carica L. (Figs 10-11).

NOTES. First record of the species from Morocco. $S$. parvula was reported previously from France and Spain. See also remarks under $S$. parvilamellata.

DIAGNOSIS. S. parvula and S. parvilamellata are close to $S$. lorifera, differing from the latter in non-thickened antennal stylus and relatively short apicoventral epandrial lobe. $S$. parvula differs from $S$. parvilamellata in antennal stylus with basal segment distinctly longer than apical segment; cercus straight, black, with equally short setae; phallus narrowed distally.

\section{Sybistroma theodori Grichanov et Nourti, nom.n.} Figs 5, 7.

Hercostomus quadrifilatus Becker, 1917: 198 (in key), 234, Figs 55, 56 (nec Strobl, 1899). Type locality: not given (Spain).

Sybistroma quadrifilata (Becker, 1917) (nec Strobl, 1899), comb.n.

MATERIAL EXAMINED. Neotype $\sigma^{7}$, here designated (dried from ethanol and mounted on pin): Morocco, Larache Province,

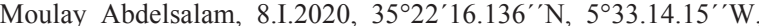
649 m, K. Kettani leg. (ZIN)

HABITAT. The habitat represents a moorland covered with Erica sp. and Cistus sp. and dotted with rocks on a


8 limestone ground (Fig. 12). A scrubland of Quercus suber surrounded the moor.

NOTES. First record of the species from Morocco. It is unclear, why did Theodor Becker [1917] describe a new species under the name "quadrifilatus Strobl". He did not provide any data on the material examined. We can only suppose that this material originated from Spain. Both Stackelberg [1934] and Parent [1938] included the species into their keys of Hercostomus, re-published Becker's description and figures, but adding no new material and points of distribution. Morge and Negrobov [1981] placed G. quadrifilatus Strobl in synonymy with $H$. parvilamellatus and noted that the Becker's description deserved a new name. None specimen with the name quadrifilatus was found in European museums keeping Becker's collections, being most probably lost. Therefore, we designate here the neotype of Hercostomus quadrifilatus Becker, 1917 collected in Morocco. The specimen corresponds to the original description by Becker [1917] and to the diagnoses provided in keys by Stackelberg [1934] and Parent [1938]. The morphology of the species corresponds entirely with the generic concept of Sybistroma as described by Brooks [2005]. The antenna of its male has enlarged scape, reduced pedicel, arista-like stylus with one or more lamellae; the hypopygium has elongated and setose apicoventral epandrial lobe, and basiventral epandrial lobes are elongated and digitiform, shifted ventrally and lying beside hypandrium; the wing has weak sinuous anterior bend before middle.

ETYMOLOGY. The species name is dedicated to famous German dipterist, Theodor Becker (1840-1928).

DIAGNOSIS. The species keys to Sybistroma spectabilis (Parent, 1928), which has postpedicel mostly yellow-orange, rounded. S. theodori has entirely black antenna with angular apex of postpedicel [see also figures in Becker, 1917; Stackelberg, 1934; Parent, 1938].
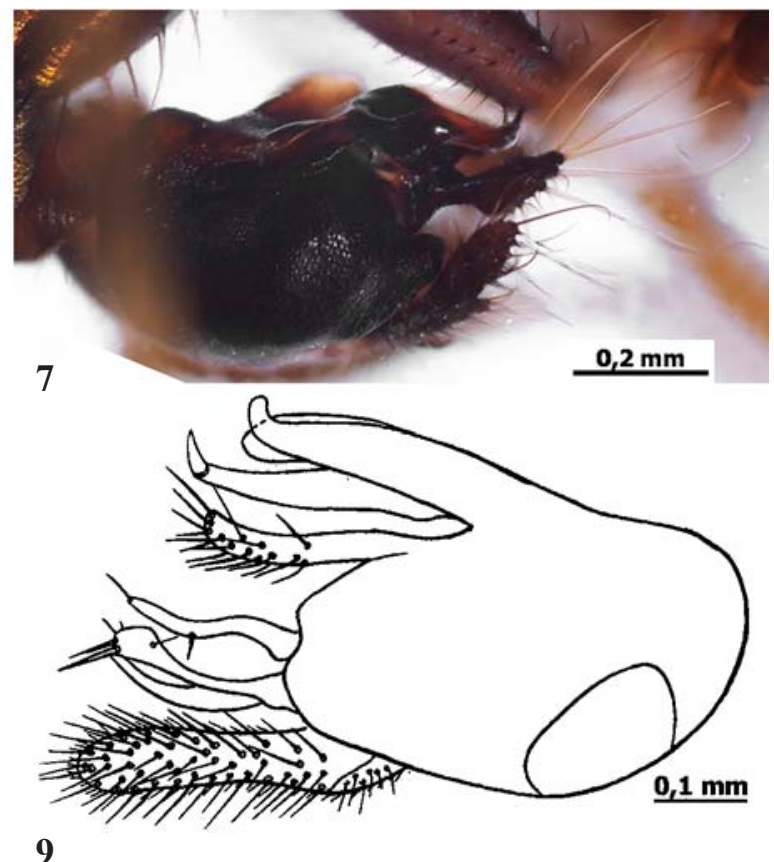

Figs 6-9. Hypopygium: 6 - Ortochile morenae; 7 - Sybistroma theodori; 8-9 - Sybistroma parvula; 6, 8, 9 - left lateral view; 7 - right lateral view. Photographs by I.Ya. Grichanov; 9 - after Morge, Negrobov, 1981.

Рис. 6-9. Гипопигий: 6 - Ortochile morenae; 7 - Sybistroma theodori; 8-9 - Sybistroma parvula; 6, 8, 9 - слева; 7 - справа. Фото И.Я. Гричанова; 9 - по Morge, Negrobov, 1981. 


\section{Key to the Mediterranean SPeCies of Sybistroma (Males)}

This key is built on Grichanov, Kazerani [2014] but has modifications based on material examined for the present study. The species distribution follows the same work.

1. At least femora entirely or almost entirely brown-

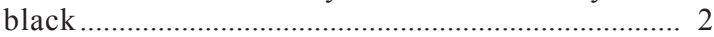
— Legs mainly yellow or reddish yellow ...................... 11

2. Antennal stylus very long, basodorsal, with apical flag .... 3

- Antennal stylus simple ................................................. 6

3. Antennal stylus with rounded or ovate apical flag, flag entirely black; fore and mid tibiae yellow or red-yellow, at least basal part of hind tibia yellow or reddish ........... 4

- Antennal stylus with lanceolate apical flag, flag black with white apex; legs entirely or almost entirely brownblack

4. Antennal postpedicel mostly yellow-orange, rounded; cercus obovate, 1.5 times longer than wide; body length $3.75 \mathrm{~mm}$ (France and Spain) .....

S. spectabilis (Parent, 1928)

- Antenna entirely black with angular apex of postpedicel; cercus band-like, about 3 times times longer than wide; body length $3.0 \mathrm{~mm}$ (Morocco and Spain)

S. theodori Grichanov et Nourti, nom.n.

5. Antennal stylus with apical expansion of first article in addition to apical flattening of second article; body length 3.5 mm (Hungary and Romania) ...... S. maerens Loew, 1873

- Stylus with apical flattening only; apical flag of stylus rhomboid, black, white at extreme apex; body length 3.5 $\mathrm{mm}$ (the Caucasus and Turkey).....

S. transcaucasica (Stackelberg, 1941)

6. Antennal postpedicel not longer or slightly longer than high.....

- Postpedicel at least 1.5 times longer than high at base 9

7. Antennal stylus of uniform thickness throughout; apicoventral epandrial lobe much longer than rounded-triangular cercus; body length 2.5-3.0 mm (Croatia, France, Greece, Italy, Turkey) ............... S. lorifera (Mik, 1878)

- Antennal stylus normal, tapering; apicoventral epandrial lobe shorter than band-like cercus .............................. 8

8. Antennal stylus with basal segment slightly shorter than apical segment; cercus elbowed, yellow, with long apical setae; phallus expanded distally; body length $3.0 \mathrm{~mm}$ (Belgium, France, Germany, Italy, Spain and UK) ....... S. parvilamellata (Macquart, 1827)

- Antennal stylus with basal segment distinctly longer than apical segment; cercus straight, black, with equally short setae; phallus narrowed distally; body length $3.0 \mathrm{~mm}$ (Morocco and Spain) ........... S. parvula (Parent, 1927)

9. Stylus middorsal; postpedicel 1.5 times longer than high at base; apicocentral epandrial lobe expanded distad, with very long hooked setae; body length $3.0 \mathrm{~mm}$ (from France across Europe to the Caucasus)

S. caudata (Loew, 1859)

- Stylus almost apical or subapical; postpedicel at least twice longer than high at base; epandrial lobe with short simple setae

10. Stylus shorter than postpedicel; apicoventral epandrial lobe band-like, much longer than cercus, longer than hypopygium; body length $3.0 \mathrm{~mm}$ (West, Central and South Europe) ........................ S. inornata (Loew, 1857)

- Stylus longer than postpedicel; apicoventral epandrial lobe much shorter than cercus and hypopygium; body length 3.3-3.5 mm (Golan Heights, Iran, Israel, Turkey) ............. S. occidasiatica Grichanov et Kazerani, 2014
11. Lower postocular setae white ……............................ 12

- Postocular setae entirely black ................................... 22

12. Antennal stylus very long, with apical flag ............... 13

— Antennal stylus simple ................................................. 17

13. Face densely covered with light hairs increasing in length downwards; postpedicel 11 times longer than high at base, with rounded expansion at $1 / 3$ in addition to ovoid apical flag on stylus; body length $3.5 \mathrm{~mm}$ (Israel) ........

S. israelensis (Grichanov, 2000)

— Face glabrous; postpedicel much shorter .................. 14

14. Postpedicel twice longer than high at base ............... 15

- Postpedicel not longer than high ................................. 16

15. Fore tarsus modified, basitarsus with narrow apical projection bearing 4 long cilia, tarsomere 2 with a short anterior and a long posterior seta at apex, 3rd tarsomere as long as 1 st and 2nd combined; mid femur with long fine ventral setae; antennal stylus with strong, almost round, black flattening at apex; body length $5.0 \mathrm{~mm}$ (Austria, France, Italy, Portugal, Spain) ................. S. eucera (Loew, 1861)

- Fore tarsus simple; mid femur without long setae; antennal stylus with long and rather narrow apical widening that is black in basal half and white in apical half; body length $3.0 \mathrm{~mm}$ (Bulgaria, Greece, Hungary, Israel, Italy, Romania, Russia (Krasnodar), Turkey) S. impar (Rondani, 1843)

16. Antennal stylus with spatulate apical flag with white narrow apical half; mid and hind femora with yellow basoventral setae; body length $3.9 \mathrm{~mm}$ (Turkey) ............ S. schachti Naglis, 2011

- Antennal stylus with rounded black apical flag with short whitish pointed apex; mid and hind femora bare ventrally; body length $3.5 \mathrm{~mm}$ (South Europe, North Africa, the Caucasus) ......................... S. dufouri Macquart, 1838

17. Fore tarsus simple; hypopygium mostly yellow; antennal postpedicel 1.33 as long as high; stylus much longer than pedicel, located before middle of dorsal surface ...... 18

- Fore tarsus modified; other features variable ............ 19

18. Abdomen thin; segment 7 long and thin; hypopygium reaching posterior margin of abdominal segment 2; body length 3.5-4.0 mm (Europe except North eastward to Novgorod Region and Mordovian Republic of Russia, Turkey, the Caucasus) ..... S. obscurella (Fallén, 1823)

- Abdomen thick; segment 7 short and thick; hypopygium reaching middle of abdominal segment 4 ; body length 3.0 mm (Afghanistan, Belgium, Czech, Hungary, Italy, Slovakia) ................................... S. sciophila (Loew, 1869)

19. Antennal postpedicel not longer than high; stylus of uniform thickness throughout, with apical article 5 to 6 times as long as basal; foreleg with basitarsus bearing row of long ventral setae, tarsomere 5 white, enlarged and laterally compressed; body length $3.75-4.75 \mathrm{~mm}$ (from France across Europe to the Caucasus and Iran) ............. S. crinipes Staeger, 1842

- Postpedicel at least 1.5 times longer than high; stylus normal, tapering, with apical segment at most 2.5-3 times as long as basal one; foreleg not as above ................ 20

20. Foreleg with tarsomere 5 flattened and black, greatly enlarged, tarsomere 4 short and slightly broadened; antennal stylus mid-dorsal; body length $3.5-5.5 \mathrm{~mm}$ (from France across Europe to the Caucasus and Iran) ........... S. discipes (Germar, 1821)

Foreleg with tarsomere 5 white

21

21. Tarsomere 4 and 5 of foreleg moderately enlarged; antennal stylus middorsal, with dot-like thickening at middle; body length $4.5-5.0 \mathrm{~mm}$ (Georgia and Iran)

S. clara (Negrobov et Onishchenko, 1991)

- Tarsomere 5 of foreleg slightly enlarged; antennal stylus 
basodorsal; body length $4.0 \mathrm{~mm}$ (Central and South Europe, Turkey) .............. S. sphenoptera (Loew, 1859)

22. Legs simple; antennal stylus with black subapical flattening; postpedicel more than 4 times as long as high; cercus simple; body length $3.0 \mathrm{~mm}$ (Austria, Bulgaria, Hungary, Romania, Slovakia).................. S. setosa Schiner, 1862

- Fore or mid legs modified; other features various .... 23

23. Wing with deep emargination between $\mathrm{M} 1+2$ and $\mathrm{CuA} 1$; antennal scape and pedicel entirely black; mid tarsus simple; cercus simple, subrectangular; body length $3.9-4.4 \mathrm{~mm}$ (Egypt and Israel) ........... S. sinaiensis (Grichanov, 2000)

- Wing simple; mid tarsus modified; other features various

24. Tarsomeres 3-5 of midleg widened and flattened laterally, black; antennal scape and pedicel yellow ventrally; body length $3.1 \mathrm{~mm}$ (Israel and Golan Height)

S. golanica (Grichanov, 2000)

- Tarsomeres 3-5 of midleg somewhat widened, black, and tarsomere 5 snow-white; antennal scape yellow ventrally; body length $3.0-4.0 \mathrm{~mm}$ (West, Central and South Europe, Egypt, Iran, Iraq, Turkey).

S. nodicornis Meigen, 1824

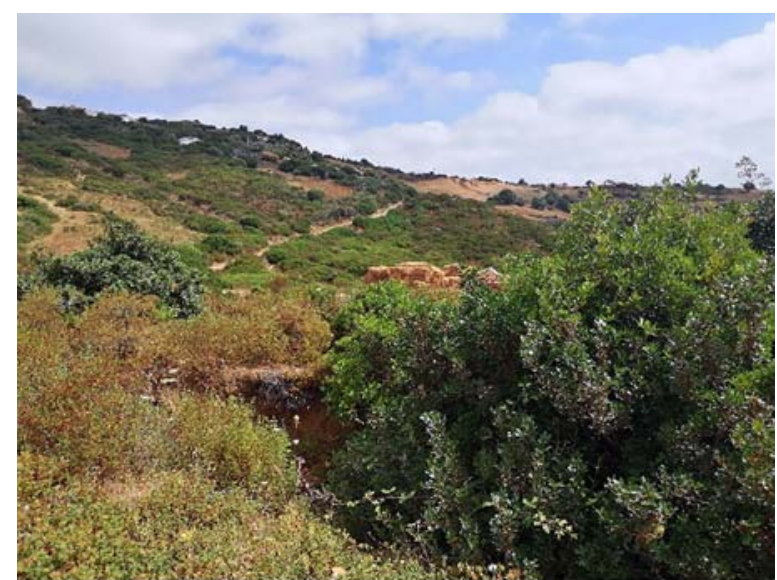

10

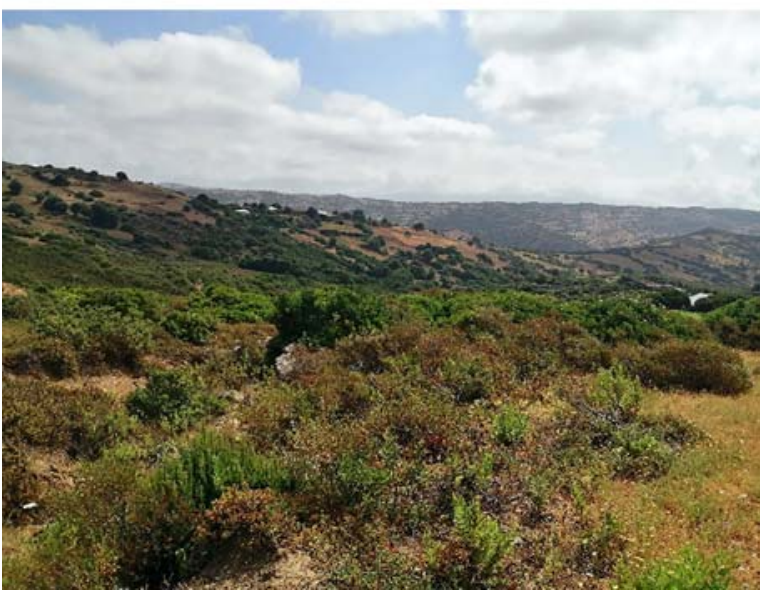

11

Figs 10-11. Fahs-Anjra locality of Sybistroma parvula (Parent, 1927) and Ortochile morenae (Strobl, 1899) (10) and surrounding landscape (11). Photographs by M. Nourti, 15.II.2020.

Рис 10-11. Местообитания Sybistroma parvula (Parent, 1927) и Ortochile morenae (Strobl, 1899) в провинции Фахс-Анжра (10) и окружающий ландшафт (11). Фото М. Ноурти, 15.II.2020.

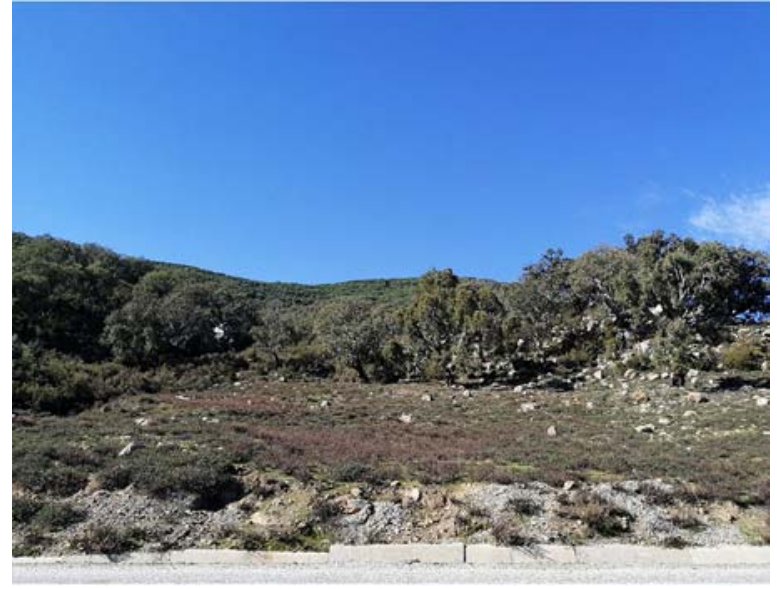

Fig. 12. Moulay Abdelsalam type locality of Sybistroma theodori Grichanov et Nourti, nom.n. in Larache Province. Photograph by courtesy of K. Kettani, Université Abdelmalek Essaâdi, Tetouan, Morocco, 8.I.2020.

Рис. 12. Типовое местообитание Sybistroma theodori Grichanov et Nourti, nom.n. около Мулай Абдельсалам в провинции Лараш. Фото К. Кеттани, Университет им. Абдельмалека Эссаади, Тетуан, Марокко, 8.I.2020.

\section{Teuchophorus rifensis Nourti, Grichanov et Kettani, 2019}

Teuchophorus rifensis Nourti, Grichanov, Kettani, 2019b: 106. Type locality: Morocco: Rif, Chefchaouen, Oued Souk Lhad.

Teuchophorus bipilosus Vaillant, 1952: 8, Fig. 17; Negrobov et al., 1984: 41, Fig. C (nec Becker, 1908, misidentification).

NOTES. Studying the papers of Vaillant [1952] and Negrobov et al. [1984], we have found a similarity of the hind tibia on photo of our T. rifensis and that on line drawings of T. bipilosus Becker, 1908 in those papers. Grootaert et al. [1995] studied the T. bipilosus types from the Canary Islands, found them corresponding with their material from France, and supposed that the Russian specimen [Negrobov et al., 1984] belonged to an undescribed species. We have reexamined the $T$. rifensis holotype and found a place of the second broken seta on both left and right hind tibiae above the pictured long ventral seta. We conclude here that T. rifensis is also distributed in Algeria and Russian Krasnodar Territory in addition to Morocco. T. bipilosus was also reported from Madeira and Iberian mainland, but association of these records is unclear at present. The two species can be distinguished as follows:

1. Hind tibia with one fine and long ventral bristle, $3-3.5$ times as long as diameter of tibia, and one very long ventral bristle, $4-5$ times as long as diameter of tibia; adjacent hairs distally about as long as diameter of hind tibia; body length $2 \mathrm{~mm}$ (Algeria, Morocco, Krasnodar Territory of Russia) T. rifensis Nourti, Grichanov et Kettani, 2019

- Hind tibia with 2 equally long ventral bristles, 4-5 times as long as diameter of tibia; adjacent hairs distally about two times as long as diameter of hind tibia; body length $1.8 \mathrm{~mm}$ (France, Portugal, Spain)...... T. bipilosus Vaillant, 1952

Acknowledgements. The authors are sincerely grateful to Dr. Kawtar Kettani (Université Abdelmalek Essaâdi, Tétouan) for her kindness in commenting on earlier drafts of the manuscript. The work of Igor Grichanov was supported by the All-Russian Institute of Plant Protection project No 06652019-0014. 
Competing interests. The authors declare no competing interests.

\section{References}

Becker T. 1917. Dipterologische Studien. Dolichopodidae A. Paläarktische Region // Nova Acta Academiae Caesareae Leopodinisch-Carolinae Germanicae Naturae Curiosorum. Vol.102. No.2. P.113-361.

Bezzi M. 1903. Orthorrhapha Brachycera // Becker T., Bezzi M., Bischof J., Kertész K., Stein P. (Hrsg.). Katalog der paläarktischen Dipteren. Bd.2. S.1-396.

Brooks S.E. 2005. Systematics and phylogeny of the Dolichopodinae (Diptera: Dolichopodidae) // Zootaxa. Vol.857. P.1-158.

Cumming J.M., Wood D.M. 2017. Adult morphology and terminology // Kirk-Spriggs A.H., Sinclair B.J. (eds.). Manual of Afrotropical Diptera. Nematocerous Diptera and lower Brachycera. Suricata 4. Pretoria: SANBI Graphics and Editing. Vol.1. P.89-134.

Grichanov I.Ya. 2007. A checklist and keys to Dolichopodidae (Diptera) of the Caucasus and East Mediterranean // Plant Protection News Supplement. VIZR RAAS, St. Petersburg. P.1160.

Grichanov I.Ya., Kazerani F. 2014. A new species of Sybistroma Meigen (Diptera: Dolichopodidae) from the Middle East with a key to West-Palaearctic species of the genus // Zootaxa. Vol.3866. No.4. P.572-582.

Grichanov I.Ya., Brooks S.E. 2017. Dolichopodidae (longlegged dance flies) // Kirk-Spriggs A.H., Sinclair B.J. (eds.). Manual of Afrotropical Diptera. Nematocerous Diptera and lower Brachycera. Suricata 5. Pretoria: SANBI Graphics and Editing. Vol.2. P.1265-1320

Grootaert P., Stark A., Meuffels H.J.G. 1995. Notes on Mediterranean Teuchophorus species with the description of a new species from the nature reserve of El Kala, north east Algeria (Diptera, Empidoidea, Dolichopodidae) // Bulletin de 1'Institut Royal des Sciences Naturelles de Belgique, Entomologie. Vol.65.
P.109-113.

Macquart J. 1827. Platypézines, Dolichopodes, Empides, Hybotides (Diptera) // Insectes Diptères du Nord de la France. Vol.3. P.1159.

Morge G., Negrobov O.P. 1981. Über die Typen von P. Gabriel Strobl aus der Familie Dolichopodidae (Diptera) // Beiträge zur Entomologie. Bd.31. S.13-15.

Negrobov O.P., Grichanov I.Ya., Shamshev I.V. 1984. [A review of Palearctic species of the genus Teuchophorus Loew (Dolichopodidae, Diptera)] // Nauchnye Doklady Vysshei Shkoly. Biologicheskie nauki. No.9. P.37-42 [in Russian].

Nourti M., Grichanov I.Ya., Kettani K. 2019a. New records of longlegged flies (Diptera, Dolichopodidae) from Morocco // Acta Biologica Sibirica. Vol.5. No.3. P.118-130.

Nourti M., Grichanov I.Ya., Kettani K. 2019b. A new species of Teuchophorus Loew, 1857 (Dolichopodidae, Diptera) from Morocco // Nature Conservation Research. Vol.4. No.4. P.106-110.

Parent O. 1927. Les espèces paléarctiques du genre Hercostomus Lw // Annales de la Société Entomologique de France. Vol.96. P.209-232.

Parent O. 1928. Contribution à la faune diptérologique de 1'Espagne Dolichopodidae// Junta de Ciencies Naturales Barcelone. Vol.11. No.3. P.5-32.

Parent O. 1938. Diptères Dolichopodidés // Faune de France. L'Académie des Sciences de Paris. Vol.35. P.1-720.

Stackelberg A.A. 1934. Dolichopodidae // E. Lindner (Hrsg.). Die Fliegen der Paläarktischen Region. Bd.4. H.5. S.129-176.

Stackelberg A.A. 1941. Dolichopodidae//Lindner E. (Hrsg.). Diptera. Die Fliegen der Palaearktischen Region. Bd.4. H.5. S.177-224.

Strobl G. 1899. Spanische Dipteren. IV // Wiener Entomologische Zeitung. Bd.18. H.4. S.117-128.

Vaillant F. 1952. Quelques Dolichopodidae de la zone paléarctique (Diptera) // Bulletin de 1'Institut Royal des Sciences Naturelles de Belgique. Vol.28. No.65. P.1-15.

Yang D., Zhu, Y., Wang M., Zhang L. 2006. World Catalog of Dolichopodidae (Insecta: Diptera). Beijing: China Agricultural University Press. Vol.1. P.1-704. 